\title{
Investigating physical solutions in the architectural design of distributed ship service systems
}

\author{
Colin P. F. Shields ${ }^{\mathrm{a}}$, Douglas T. Rigterink ${ }^{\mathrm{a}}$, David J. Singer ${ }^{\mathrm{a}}$ \\ ${ }^{a}$ University of Michigan, United States
}

\begin{abstract}
The design of distributed ship service systems, or distributed systems, integrates concepts of vessel layout, system functionality, and the distributed systems configuration. Understanding the design relationships between these concepts is a critical aspect of investigating and developing design requirements. Thus, in the design of complex naval vessels, the distributed systems configuration, called the physical solution, must be considered in early-stage design activities to ensure that emergent functional requirements are achievable and affordable. To address this, we propose a novel perspective for modeling and investigating physical solutions in the architectural design of distributed ship service systems. Our approach uses scalable network representations of vessel layout and functional relationships within systems to stochastically generate ensembles of distributed system solutions. Ensembles are then evaluated to determine system characteristics, bringing physical solution information into early-stage requirement elucidation. We demonstrate our approach using concept-level vessel knowledge to identify distributed system characteristics, and show the method's usefulness in understanding complex distributed systems design relationships.
\end{abstract}

Keywords:

Distributed ship service systems architecture, network theory, early-stage ship design, distributed system analysis 


\section{Introduction}

Advances in technology onboard naval vessels promise improved capability, mission effectiveness, survivability, and fleet support $[1,2]$. Realizing these benefits requires understanding how technologies and their supporting systems integrate into the overall vessel design [3, 4]. Design decisions affecting physical system integration pose significant implications for vessel vulnerability $[5,6,3,7]$, producibility $[8,9]$, cost $[10,11]$, and other performance characteristics $[12,13,14,15]$. Providing insight into how early-stage layout and functional requirements affects these vessel characteristics can help inform related decision making processes [16]. However, this requires considering the physical configuration of distributed systems within the vessel during the formative stages of requirements elucidation $[17,18]$. In this paper, we demonstrate how network representations and stochastic solution configuration can deliver new insight into the early-stage design relationship between vessel layout, functional requirements, and distributed systems characteristics.

To facilitate our study, we describe the architectural design of naval distributed systems in terms of the structure and interactions of its physical architecture, logical architecture, and operational architecture ${ }^{1}$. Physical architecture defines the spatial information about the vessel, such as component locations, space definitions, and space adjacencies. Logical architecture defines the macroscopic functional relationships between components within the vessel (e.g. a generator supplies power to an electric motor). Operational architecture defines the purpose of the system in time - how parts of the vessel and its systems are used for a given scenario.

Here, we will focus on the interaction between the physical and logical architectures, which we call the physical solution. The interaction defines the physical description of components and distributed system routings between components. The physical solution is the physical definition of the distributed system, which implements the logical architecture within the physical architecture. While the physical solution does not consider the op-

\footnotetext{
${ }^{1}$ This description reflects the findings of an ongoing research program studying the design of naval distributed systems. Research collaborators include students, researchers, and faculty at The University of Michigan, Virginia Polytechnic Institute and State University, The University College London, and Delft University of Technology. The program is supported by the U.S. Office of Naval Research, Grant No. N00014-15-1-2752.
} 
erational architecture, it still has a critical role in vessel performance [19]. Thus, it is important to understand how decisions defining the physical and logical architectures will impact on the physical solution during early-stage design activities. However, eliciting this relationship in multirole naval vessels is a complex problem and must be addressed through the consideration of material solutions [20].

In the case of vessel characteristics dependent on physical and logical architecture, design outcomes are manifested in the physical system solution. The proposed approach provides predictive methods to develop physical solution information earlier in the design process to enable trade-off analysis and requirements decision making based on distributed systems analysis.

\section{Background}

The design and integration of distributed systems in naval vessels is traditionally a split discipline. Naval concept development employs functional modeling to create preliminary representations of required system functions and vessel layout $[21,16,4]$. Following functional modeling, the physical product is developed in increasing detail $[22,23]$. In naval design, once the physical distributed systems solution is available at a sufficient level of detail, testing and simulation are conducted to understand its overall performance characteristics $[24,6,25]$. Here, the understanding of decisions regarding the physical and logical architectures lags the initial design. The detailed physical solution is predicated on concept design decisions about vessel mission, sizing and layout. Therefore, there is little opportunity to use feedback from distributed systems performance to refine decisions until after significant cost has been committed [3, 26, 27]. Addressing this requires designers to understand how the logical and physical architectures influence the physical solution earlier in the design process.

Currently ship designers have limited options for modeling and studying physical solution outcomes in early-stage design [28, 29]. Heavy coarsegraining can reduce the system implications to parameterization, exemplified by cost estimation $[30,31]$. Parameterization-based approaches are applicable for specific relationships in the design, but requires a representative database of similar vessels to be developed. Additionally, parameterization is limiting because it only predicts the outcome of a decision without revealing the underlying distributed systems configurations which drive the design relationship. 
More recently, designers have addressed the physical solution with computeraided design (CAD) models. This provides a detailed system model that can be analyzed to validate functions and predict attributes of the physical systems [4]. Efforts in automated distributed systems design have been successful at introducing physical system solutions sooner in the ship design process $[32,33,34]$. Automated methods generate physical solution models through routing optimization [35], resource demand satisfaction [7], and system templates $[36,37]$ which are then integrated into a larger vessel model.

However, in the initial stages of concept design and requirements elucidation, automated design approaches may not be viable. From a methods perspective, CAD is used to visualize design concepts, providing new insight and enabling further solution definition and model development [38, 39]. This means that in early-stage design, the required product definition for CADbased automation has not been well defined [40]. Despite the continuous improvement of CAD tools and applications, in concept design there is still limited information available.

Even in low-fidelity applications, generation methods need specification of system components and their usage profiles. Providing the necessary physical definition and system loads requires a significant amount of design knowledge about vessel geometry, mission, and operations. The amount of required knowledge is compounded by uncertainties caused by the concurrency of naval design and component development [41, 42]. The result is that meeting geometric modeling and design definition requirements can quickly become drivers or lead to decision making based on modeling goals or constraints $[43,44,45]$.

The potential impacts of automated approaches can be seen in the earlystage ship design automated arrangement program, Intelligent Ship Arrangements (ISA). ISA enables designers to automate the rapid generation of balanced space arrangements by considering large sets of constraints including separation and adjacency requirements, preferred locations, and space shape $[46,47]$. ISA was quickly extended to explore passageway design based on lattice templates $[48,49]$. The approach produces high-quality arrangement designs, but requires significant design knowledge of space usage and relationships. This can over-constrain the solution space in early-stage design, creating dominant design outcomes and heavily biased solutions [50, 51].

Automated distributed systems design methods also struggle to address the system design relationships from a process perspective. The goal of addressing distributed systems in early-stage ship design is to understand how 
the architectural design of distributed systems affects performance. Automated approaches bias this relationship by generating physical system solutions through optimizations and system templates. Similar to parameterization, this helps predict outcomes of the relationship, but does not help explain how or why the relationship causes the outcome.

Automated approaches can produce detailed solutions to well-defined design problems at specific modeling fidelity - often optimized to an objective function. The resulting physical system solutions and characteristics are then used as a basis for decision-making. However, early in the design process of complex multirole vessels, the problem is constantly developing [52] and defining an appropriate objective function is problematic [17]. Further, the early-stage design activities have fidelity ranging from paper sketches to CAD representations of the physical product [23]. Thus, optimal solutions to fixed system objectives are poor grounds for understanding the possible design relationships. Instead, an early-stage approach to physical system solutions must be independent of an objective function.

Network-based methods and tools have previously proven effective for understanding interdependent relationships in naval design without relying on optimization or simulation. Network analysis studies the evolving interactions between parts of the design process and product [53]. This allows designers to represent and analyze disparate information at multiple scales throughout the design process [19]. For example, studying the interdependent coupling between arrangement constraints and physical architectures can identify optimization biases and predict vessel arrangements [50, 51]. Network analysis can also enable designers to identify design drivers built into the structure of naval architecture methods and tools [53, 54, 55]. Applied to studying physical system solutions, network-based methods provide insight into distributed systems functionality and design [19, 18, 14, 15].

In this paper, therefore, we focus on understanding the interdependent design relationship between logical architecture, physical architecture, and physical system solutions with a scalable and stochastic network method. This requires approaching physical system solutions in terms of its feasible configurations, not its optimal configurations. Network representations are used with stochastic system routing methods to generate feasible configurations of the physical system solution. Large sets of configurations are then evaluated to probabilistically explore the possible design relationships. The approach formalizes previous network-based distributed systems representations and is a unique perspective on system generation. Further, this method 
is a promising step towards better understanding the architectural design of distributed systems in naval vessels.

The remainder of this paper is structured as follows: a network representation of physical and logical architectures is discussed. The physical solution generation method is presented and a notional vessel is used to demonstrate the method. The paper concludes with a discussion of results and future work.

\section{Methodology}

In this section, a short review of network theory is provided followed by a description of the methods used in this paper. Then an application of the proposed methods is presented.

\subsection{Vessel and System Network Representations}

Network based representations of vessels and systems have been shown to enable novel analysis and synthesis methods while requiring minimal designer input outside of traditional early-stage ship design activities. Efforts to introduce physical architecture, logical architecture, and distributed systems physical solutions in concept design have employed network representations to model geometric structure $[50,56,57]$ and systems $[12,18,15]$. Networks, or graphs, are a collection of objects, called nodes, and the connections between them, called edges [58].

Network theory is a broad discipline that studies the structure of networks to help understand the behavior of the system of objects they represent $[58,59]$. This paper uses simplex and multiplex networks to represent vessel geometry and system connectivity respectively. Figure 1a is an example of a simplex network and its adjacency matrix $A$, the mathematical definition of that network. The adjacency matrix is an $[n \times n]$ matrix where $n$ is the number of nodes in the network. The elements of $A$ denote the network edges: $A_{i j}=1$ if an edge exists between node $i$ and $j$, otherwise $A_{i j}=0$. In this case, the nodes of the network cannot have self-edges and edges are reciprocal so $A_{i i}=0$ for all $i$ and $A$ is symmetric.

Multiplex networks, shown in Figure 1b, are a layering of the simplexes, or a network of networks. In a multiplex network with $M$ layers, each layer $l$ is an $n \times n$ adjacency matrix $A_{l}$ defining the same set of $n$ nodes [60]. Edges 


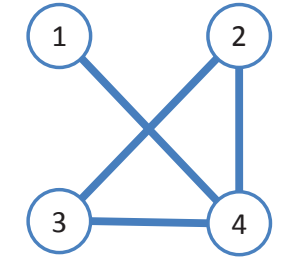

$A=\left[\begin{array}{llll}0 & 0 & 0 & 1 \\ 0 & 0 & 1 & 1 \\ 0 & 1 & 0 & 1 \\ 1 & 1 & 1 & 0\end{array}\right]$

(a) Simplex network

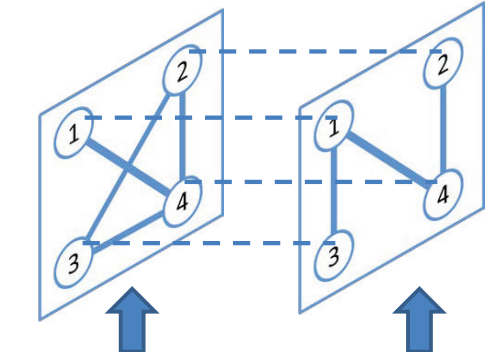

$A_{1}=\left[\begin{array}{llll}0 & 0 & 0 & 1 \\ 0 & 0 & 1 & 1 \\ 0 & 1 & 0 & 1 \\ 1 & 1 & 1 & 0\end{array}\right] \quad A_{2}=\left[\begin{array}{llll}0 & 0 & 1 & 1 \\ 0 & 0 & 0 & 1 \\ 1 & 0 & 0 & 0 \\ 1 & 1 & 0 & 0\end{array}\right]$

(b) Multiplex network

Figure 1: Examples of simplex and multiplex networks. 1a is a simplex network example and corresponding adjacency matrix $A$ where edges are denoted $A_{i j}=1.1 \mathrm{~b}$ is a multiplex network with $M=2$ layers. Nodes are the same in each layer, but the connectivity within layers, defined in $A_{i j, 1}$ and $A_{i j, 2}$, are independent of edges in the other layer.

within each layer represent a distinct type of connection between nodes. Multiplex representations provide additional information about the interdependencies between each represented system layer that would not be captured through individual simplexes.

\subsubsection{Physical Architecture Network Representation}

Networks provide a vessel representation that can be used for design analysis $[12,50,51,18]$, physical architecture generation [56, 57] and physical solution generation $[35,7]$. Though the fidelity and terminology of the cited works' network models vary, they stem from adjacencies of spatial modeling units such as compartments, structural zones, or unit volumes.

In the network representation shown in Figure 2, each spatial modeling unit is a node in the network simplex representing a section of the vessel geometry or physical architecture. Adjacency to other units is represented by edges. The network representation is either created by the designers or extracted from a vessel arrangement concept. Once created, it acts as a surrogate for precise geometric definitions that does not require extensive hullform and compartment modeling. This allows the network to model the vessel at scalable levels of design detail by altering the density of the network 


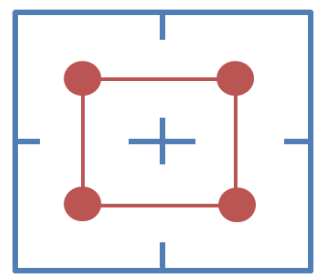

(a) Network

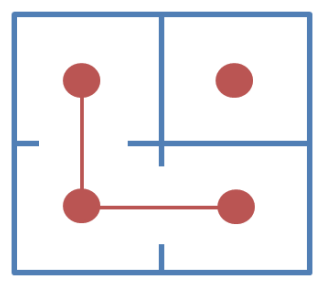

(b) Edge removal

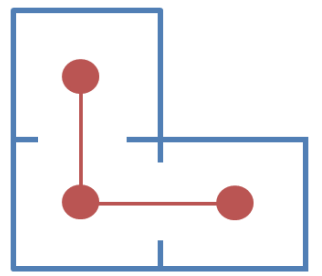

(c) Node removal

Figure 2: Demonstration of the physical architecture network representation. 2a shows four adjacent, connected compartments (blue) with the network representation overlaid (red). $2 \mathrm{~b}$ and $2 \mathrm{c}$ show the removal of connections and compartments with the corresponding network change.

and what each node represents.

Networks representing the vessel geometry can also be altered to represent changing geometry through removal of nodes or edges. This is demonstrated in Figure 2, which shows how changes in a physical arrangement of compartments are represented in a network.

In this paper, the physical architecture network only defines adjacencies, but it can be extended to other characteristics of the vessel geometry. Distance between compartments can be stored on edges. Areas or volumes can be stored on nodes and elements can be added and removed based on interdependent aspects of the design. This allows a designer to tailor the network without changing the mathematical definition and analysis processes.

\subsubsection{Logical Architecture Multiplex Network}

Logical architecture can be described in a similar manner to the geometric relationship within the vessel. In this representation, system components are represented as nodes and the intra-system connections between components are edges $[12,18,15]$. Following the convention of previous work, systems in the vessel are defined by resource (i.e. electrical power or chilled water) and components are represented as nodes within each system network.

Using the multiplex network structure, each system is represented in a separate simplex layer, which contains nodes representing all distributed system components in the vessel model. Edges within a layer denote connectivity for the represented system. For example, a node representing a generator set has edges between itself and the nodes of all components that use the electrical power it generates. Figure 3 demonstrates a two-system multiplex. 


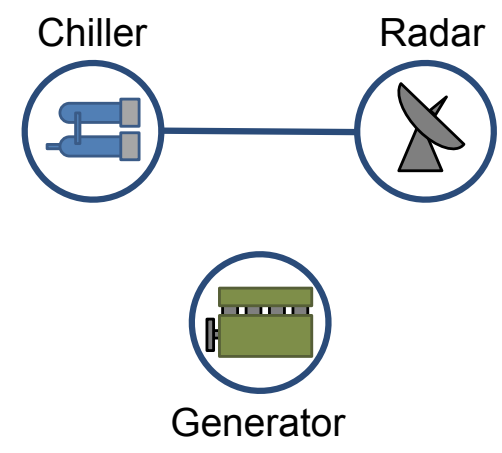

(a) Chilled water

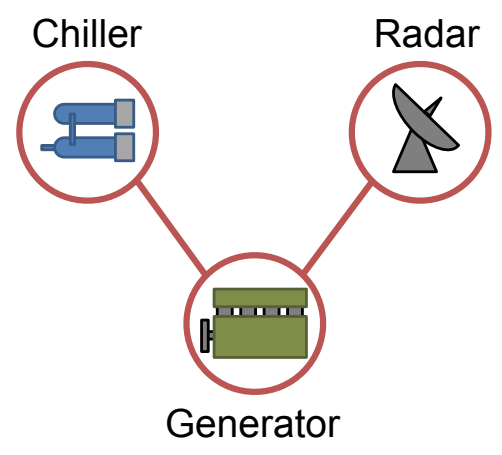

(b) Electrical power

Figure 3: Multiplex representation for chilled water (3a) and electrical power (3b) system connectivity. All modeled components are included in each simplex, but edges in a simplex are independent of other layer.

The multiplex representation requires a small amount of additional input from the designer. In concept design, the gross system components are known, but the inter-component relationships may not be. However, creating those relationships only entails a rough allocation of connectivity. Further, connectivity is often defined by other information including general zoning concepts, bulkhead locations, and component proximity.

Representing the logical architecture relationships allows the network to scale with additional components or increased component detail. For example, edges in the multiplex can be weighted to represent system loads once they are estimated by the designer. The following section presents an algorithm for converting the encoded physical and logical architectures into information about the physical solution.

\subsection{Logical-Physical Architecture Translation}

This section details a method for using the physical and logical architecture networks to stochastically generate distributed systems physical solutions. Creating physical solutions can be seen as a translation problem: Given a simplex $V$ representing the vessel's physical architecture and a multiplex $S$ representing logical architecture, the physical solution is a set of paths $G$ through $V$ that satisfy the edges in $S$. In other words, the physical solution is a realization of potential distributed systems routings through 


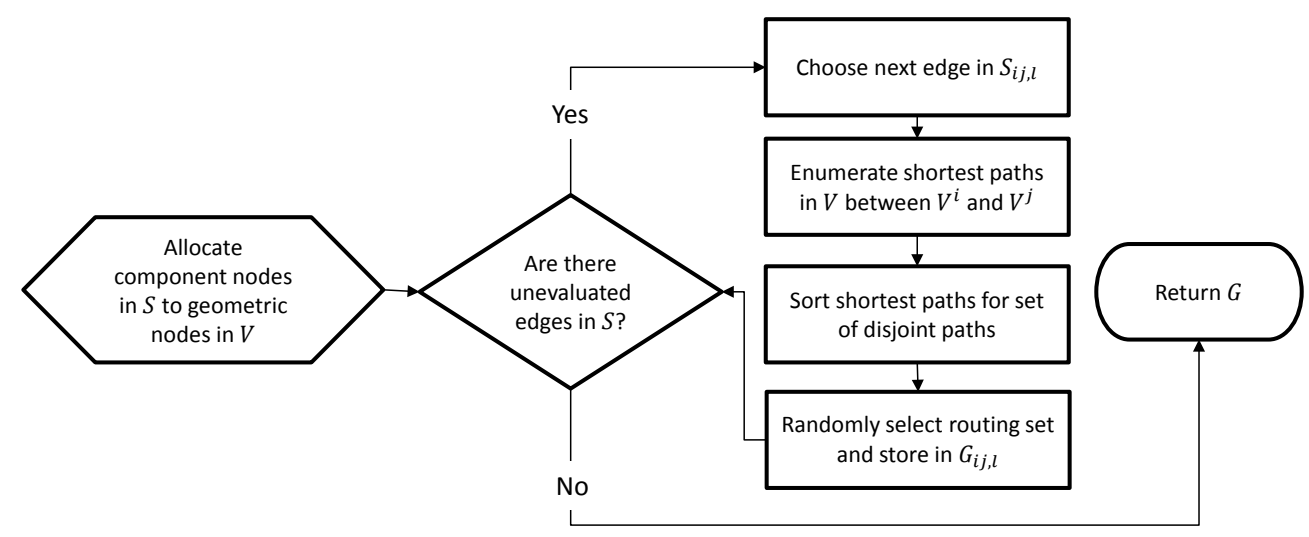

Figure 4: Process description of the logical-physical architecture translation algorithm.

the physical architecture geometric relationships that support the logical architecture connectivity. As such, resulting routings only represent feasible physical solutions, which in turn must be analyzed to determine characteristics using distributed system analyses methods (e.g. [19, 18, 15]).

The proposed Logical-Physical Architecture Translation (L-PAT) algorithm generates routing through the geometric relationships of the vessel's physical architecture $V$ that satisfy the system connectivity relationships in the logical architecture $S$. The general steps of the L-PAT algorithm are described in Figure 4.

The L-PAT algorithm is initialized by the user defining $S$ and $V$, then assigning component nodes in $S$ to location nodes $V$. This is defined as component node $i$ in $S$ mapping to a node in $V$, which is stored as $V^{i}$ and denotes where the component $i$ is located in the geometric network. Next, for each system layer $l$ and contained component connectivity edge $S_{i j, l}$, routings are found between $V^{i}$ and $V^{j}$ using sets of disjoint paths in $V$. Disjoint paths between nodes through the network share only a starting and end point. All shortest paths between $V^{i}$ and $V^{j}$ are found using Dijkstra's network based shortest paths algorithm [61].

Once shortest paths have been enumerated, they are sorted in a list of sets of disjoint paths. The routing is then randomly selected from this list. The path enumeration and routing selection process is demonstrated in Figure 5 . Selected routes are stored in an array of routing definitions $G$ where $G_{i j, l}$ is the set of edges defining the routing between components $i$ and $j$ for multiplex layer $l$. This process is completed for each edge in $S$, completing 


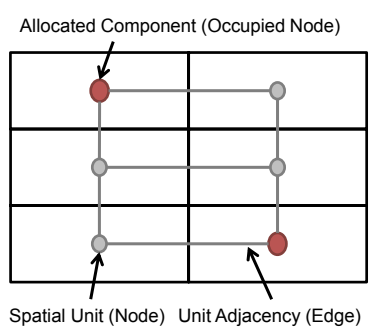

(a) Geometry network $V$ and components

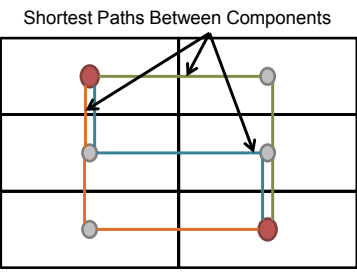

(b) Enumerated shortest paths

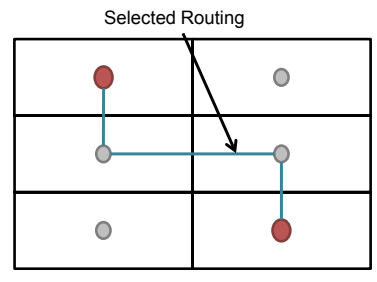

(c) Route randomly selected from paths

Figure 5: Demonstration of L-PAT algorithm route generation process. 5a is a visualization of a physical architecture network with two components which will be connected. 5b shows possible edges for the three possible shortest path routings. 5c demonstrates the random selection of a routing (blue) from the possible paths.

the architecture translation.

The proposed path selection process is a noticeable departure from current distributed system design methods. Optimization and template-based methods prescribe architectures using best-practices implemented through known designs and fitness functions. This limits the modeling and analysis perspective to a specific design problem. Stochastic generation of distributed systems physical solutions places significance on statistical characteristics of the solution. This reveals new information about how the design relationship and corresponding outcomes develop, as opposed to presenting a discrete set of possible optima.

Physical solutions generated through this method depend on the disjoint path and shortest path routing modeling assumptions. Together these assumptions control the translation behavior for a single edge in $S$. Disjoint path requirements specify the number of physical routings for each logical connection and the level of overlap for those routings. Adjusting the set size of disjoint paths scales the physical redundancy of the logical architecture. Preventing overlap supports physical redundancy, but is not an intrinsic requirement of implementing the L-PAT algorithm. Modifying the disjoint path sorting process to allow overlap at certain locations or a percentage of path length may be used to explore relaxed solutions. Disjoint set size can also be specified for specific edges in $S$ or relaxed to include multiple sizes.

Similarly, shortest path routing is a useful modeling assumption, but is 
not required for L-PAT algorithm implementation. Due to the combinatorial difficulty of enumerating all paths in the physical architecture, it becomes necessary to restrict the evaluated paths. To do this, we assume that routings between components do deviate from the shortest possible paths. This assumption successfully restricts possible routings to a computationally manageable problem that is addressed at length in the network literature [59]. However, only examining shortest paths also restricts the emergence of possible physical solutions. Relaxing the assumption can be accomplished by modifying shortest path algorithms to include paths within a specified tolerance of the shortest length paths. Studying the effect of this relaxation is a valuable area for future work, but is not examined in this paper.

In the described implementation, paths are selected at random from disjoint sets to enable sampling of the solution space. Approaching generation by fully enumerating sets of paths across all edges in $S$ is possible, but potentially prohibitively computationally expensive. This is compounded by the fact that the L-PAT algorithm does not include analysis of the physical solution, which would add to the computational effort of full enumeration. On the other hand, sampling the possible configurations can develop a reliable description of characteristics while limiting computational expense.

\subsection{Application of the L-PAT Algorithm}

In this section, the L-PAT algorithm is applied to a notional concept design, illustrating its operation and how the resulting physical solution data can be used.

Applying the L-PAT Algorithm maps the logical architecture to the physical architecture, providing a physical solution. Analyses can leverage the structure of networks $V, S$, and $G$ to develop information about design relationships between the physical architecture, logical architecture, and physical system solution. This enables designers to explore the properties of a generated configuration based on relevant decision criteria or investigated design characteristics.

Recent studies in reducing vessel costs have demonstrated a strong correlation between construction cost and production time to outfit density $[8,9]$. The studies focused on evaluated basic ship outfit density - light ship weight less ship structure weight divided by total volume [9] - which provides an estimate of uniform density over the vessel. Thus, the outfit density metric does not provide information about the distribution of outfit density through-

out the vessel. Without this, the designer can still rely on historical results 


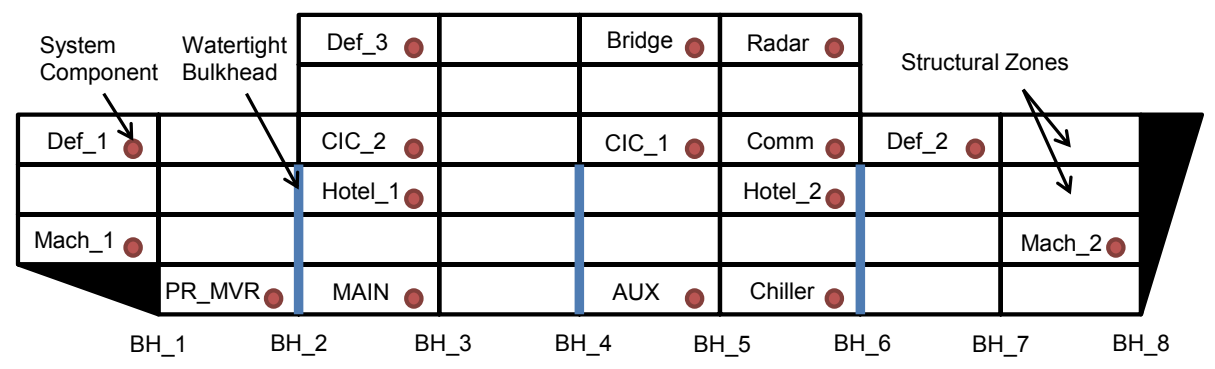

(a) Initial Vessel Configuration: Structural zone model with component locations. Abbreviations: NAME_x - component number; Def_x - defense system component; Mech_x - mechanical component; CIC_x - combat information center; Hotel_x hotel load center; PR_MVR - prime mover; Comm - communications center.
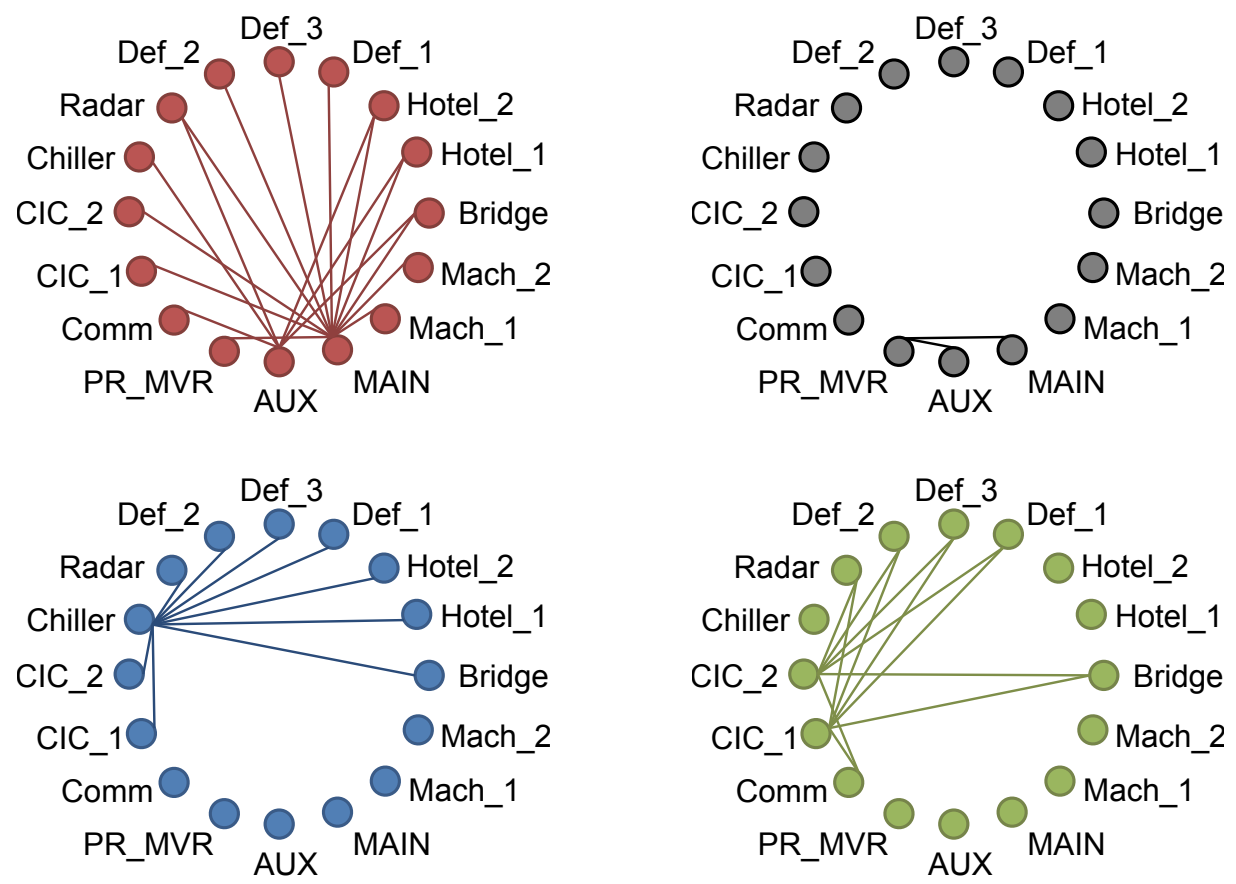

(b) System connectivities $S$ between components in logical architecture model. In order from left to right: red - power system; black - dedicated prime mover power; blue - cooling system; green - information exchange system. Personnel movement connectivity is not included, but is a fully connected network.

Figure 6: Vessel and system model for the L-PAT algorithm application cases. 
and best-practices such lengthening the vessel [62] to reduce engineering and production man-hours [9]. However, if more detailed information is available in early-stage design, designers can better understand how and why vessel arrangement and system connectivity impacts future vessel cost and the relative difficulty of detailed design and construction work.

Using the L-PAT algorithm, a basic vessel definition is used to generate a distribution of relative outfit density within the vessel. The notional vessel in Figure 6 is a structural zone model similar to those in [51]. The vessel geometry network $V$ is altered to reflect the placement of three watertight bulkheads where it is postulated that they cannot be penetrated by the physical solution routings. A two-dimensional representation is chosen for ease of demonstration and visualization of the L-PAT algorithm. However, the physical architectures can be extended to three-dimensions by adding additional nodes and edges to represent transverse zones and related adjacencies.

Following a simple logical architecture concept, logical relationships between components are identified. First, all components need electrical power from the main (MAIN) and auxiliary (AUX) generators. Specific components require chilled water for cooling and others exchange information for vessel operation. A dedicated power connection from the main and auxiliary generators to the prime mover (PR_MVR) is included for emergency operations. Finally, in this example, personnel must be able to access each component, requiring logical connections between all components in the personnel movement system. The logical architecture $S$ is shown in Figure 6b.

Figure 7 shows the network representation of the physical architecture in Figure 6 and networks for two alternative configurations. These configurations demonstrate the modeling flexibility of the proposed network perspective. The L-PAT algorithm is applied to all three configurations to show a comparative analysis of the results. Due to the 2-dimensional structural zone model and limited number of disjoint shortest paths through the physical architecture network, each logical architecture edge translation is filtered to only select single path routings. For larger or 3-dimensional zone models, this selection process can include multiple paths for each translation to better represent the potential system routing.

Distributed system routing density is evaluated as an indicator of localized outfit density. Instances of the physical solution are evaluated in an ensemble to provide the average routing density between each zone in the vessel. The average routing density $r_{i, j}$ of edge $V_{i, j}$ is defined as the average number of system types (i.e. chill water, electrical power) with a path through that 


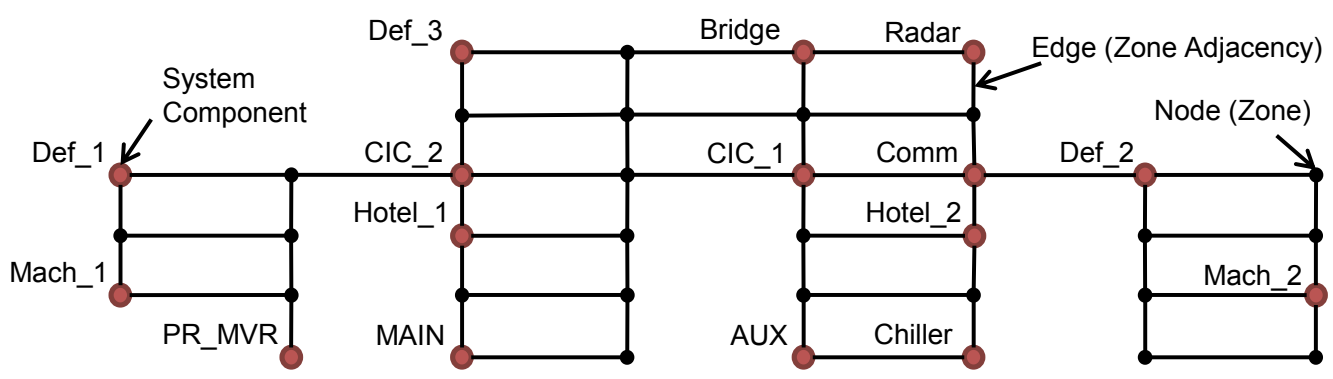

(a) Baseline Vessel: Network $V$ representing the configuration in Figure 6a. Edges between structural zones that pass through the watertight bulkheads have been removed to prevent system penetrations.

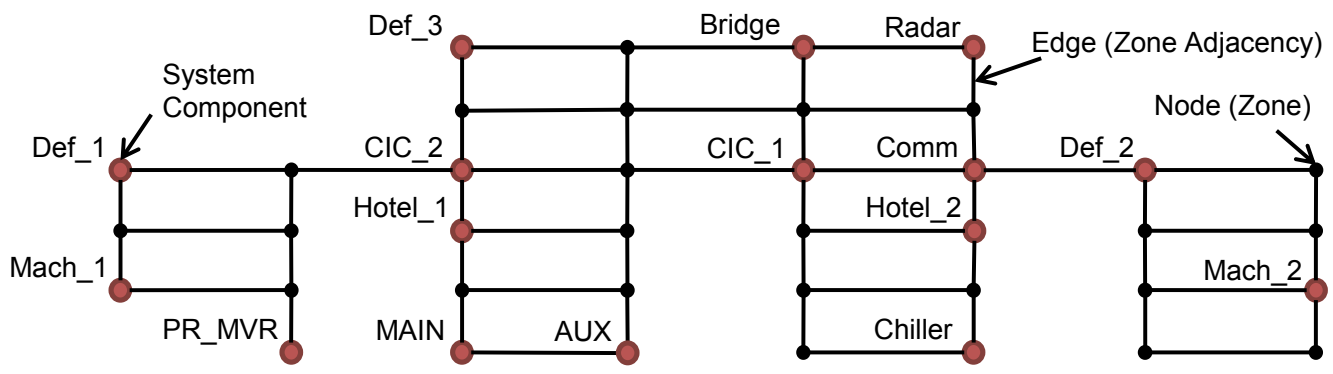

(b) Relocated AUX Configuration: Network $V$ representing a modified vessel with the AUX component moved next to the MAIN component.

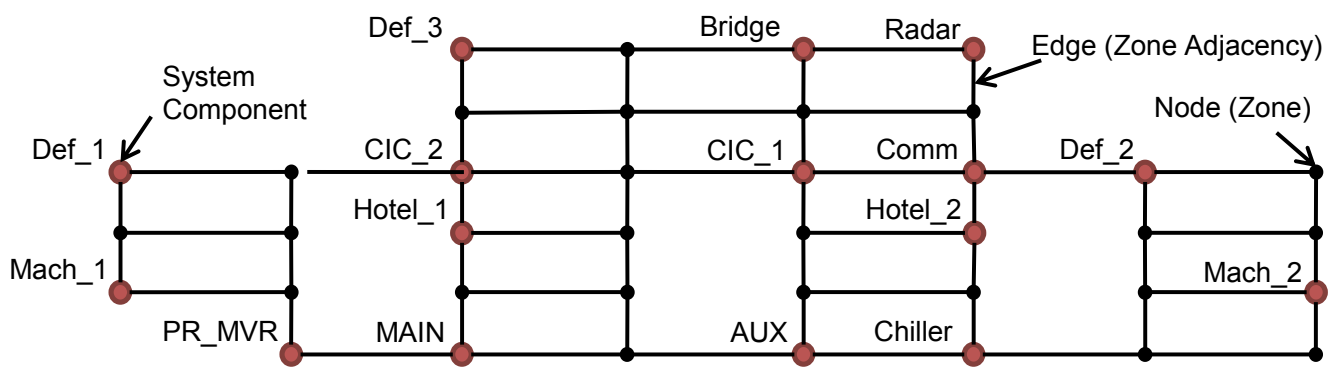

(c) Ring-bus Configuration: Network $V$ representing a vessel configuration that allows system routings longitudinally along the lowest deck.

Figure 7: Vessel models for comparative analysis with the basic vessel configuration. System connectivities $S$ are unchanged between cases. 
edge across the ensemble of physical solution instances. This calculation is given by

$$
r_{i, j}=\frac{1}{\Omega} \sum_{l=1}^{M} \delta_{i j, l}
$$

where $\Omega$ is the number instances in the ensemble, $M$ is the number of multiplex layers in $S$, and $\delta_{i j, l}=1$ if $V_{i j} \in G_{l}$ and $\delta_{i j, l}=0$ otherwise. This calculation considers the density of system types to represent a macroscopic view of system interaction within physical solutions. Analysis of interactions between edges in the logical architecture is presented in [15] and offers another application of the architecture translation perspective.

The distribution of routing density across adjacencies in the physical architecture is also analyzed. The mean routing density $\bar{r}$ and routing density variance $\sigma_{r}$ in the vessel are the expected value and variance of $r_{i, j}$ over all edges, respectively. The metrics $\bar{r}$ and $\sigma_{r}$ indicate how routing density is distributed through the vessel. Lower $\bar{r}$ values suggests systems routing in the vessel are separated - different system layers in $S$ do not share routing paths reducing outfit density. Conversely, higher $\bar{r}$ values indicate different systems are likely to share routings, increasing outfit density. Routing density variance $\sigma_{r}$ measures the spread of routing density. Lower variance suggests $r_{i, j}$ is clustered around $\bar{r}$, or the routing density is evenly distributed throughout

the vessel. High variance indicates $r_{i, j}$ is more widely spread around $\bar{r}$ and there areas of relatively high and low routing density.

\section{Results and Discussion}

The L-PAT algorithm was used to create 50 physical solutions for each vessel configuration in Figure 7. For each configuration's ensemble of physical solutions, Equation 1 was used to assess routing density on each edge. Figures $8 \mathrm{a}-8 \mathrm{c}$ show the ensemble results of the average routing density analysis. This section presents and discusses these results, starting with the baseline configuration in Figure 7a.

\subsection{Baseline Configuration}

In Figure 8a, the distribution of $r_{i, j}$ represents a conventional naval vessel physical solution. High routing densities appear in the vertical paths from the main and auxiliary power components and in a longitudinal layer above 


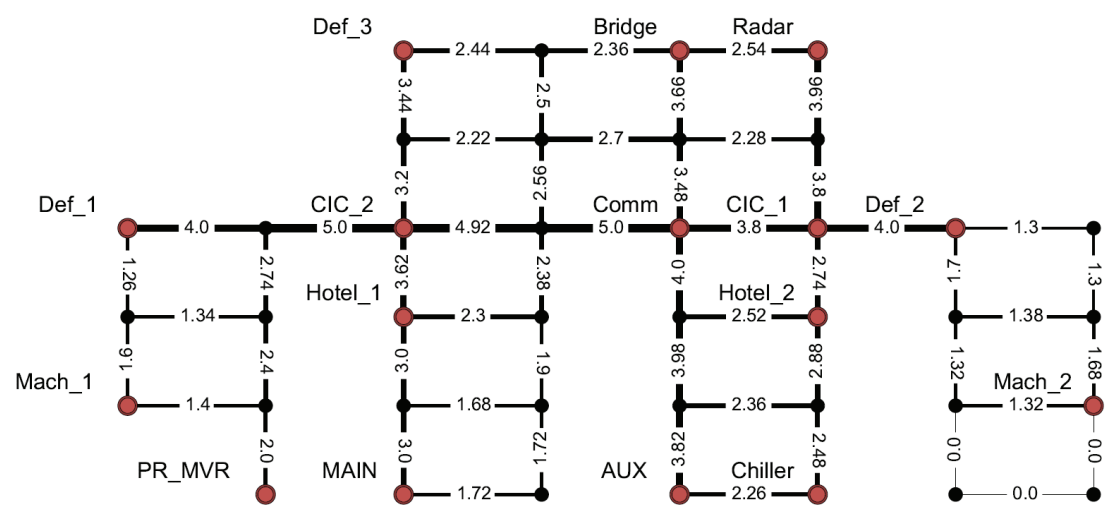

(a) Routing density results for Baseline Configuration; $\bar{r}=2.17, \sigma_{r}=1.40$

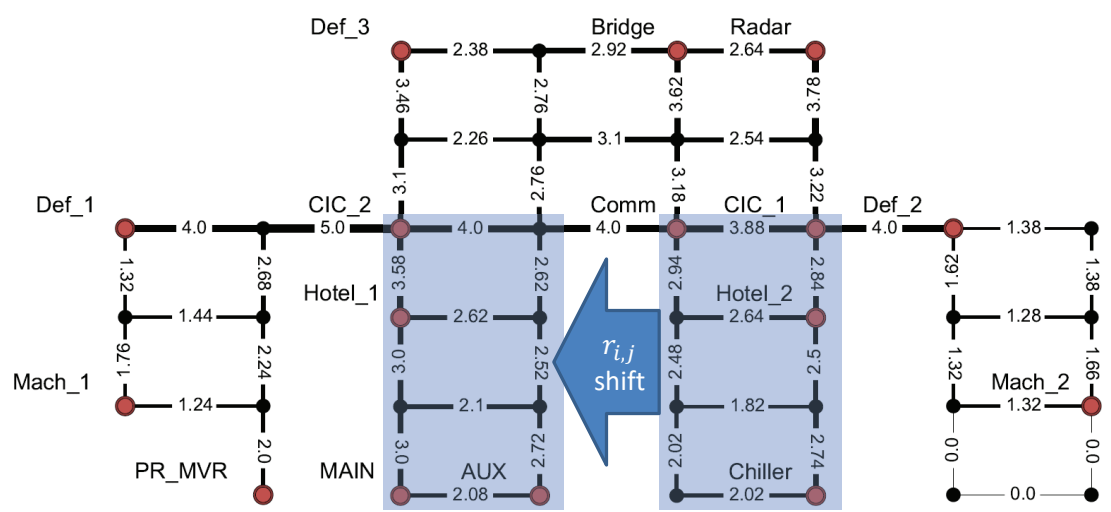

(b) Routing density results for Relocated AUX Configuration; $\bar{r}=2.11, \sigma_{r}=1.29$.

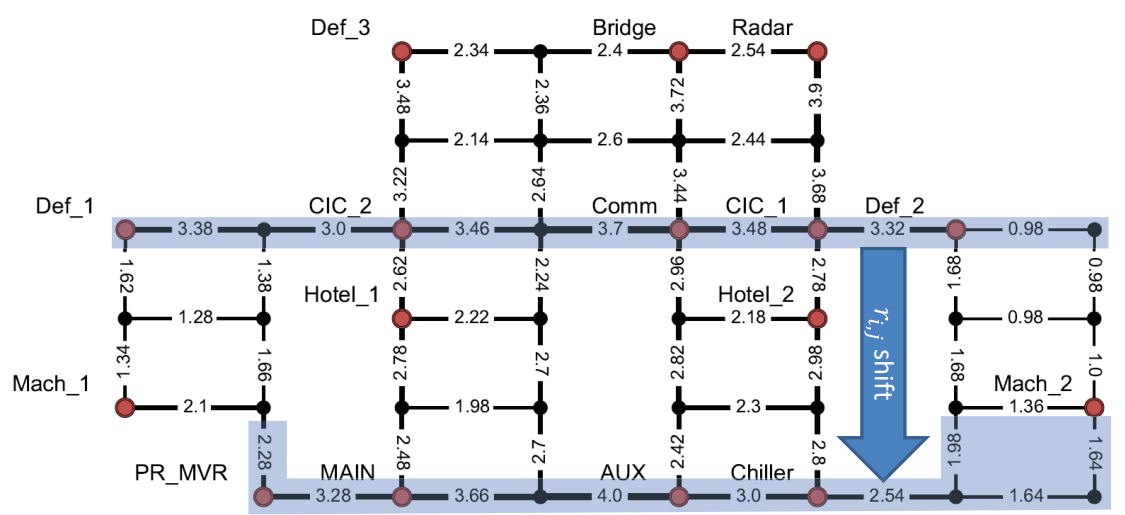

(c) Routing density results for Ring-Bus Configuration; $\bar{r}=2.49, \sigma_{r}=0.81$.

Figure 8: Distributed systems routing density $r_{i, j}$ in each vessel configuration. Regional density shifts compared to the Baseline Configuration, 8a, are highlighted in blue. 
the damage control deck. The highest possible routing densities, $r_{i j}=5.0$, are located directly above the watertight bulkheads at $\mathrm{BH} \_2$ and $\mathrm{BH} \_4$, which separate the three components that are the only ones with edges in the dedicated prime mover power system (black in Figure 6b). The fore and aft regions below the damage control deck are the lowest density regions due to the low number of components and limited system connectivities.

Analysis results of the vessel in Figure 7a demonstrate that the L-PAT algorithm can generate reasonable physical solutions given early-stage vessel design information. Additionally, the analysis shows the benefit of a probabilistic approach to realizing the distributed system design relationships. Statistical ensemble analysis helps account for uncertainty of future design decisions and lack of early-stage modeling fidelity. As opposed to automated distributed systems generation and optimization, which present defined "optimal" design outcomes, the translation method allows designers to consider how and why the design relationships may develop over the course of the design process. This is further illustrated by a comparison of results for the baseline configuration and a configuration with a moved AUX component, Figures $7 \mathrm{a}$ and $7 \mathrm{~b}$ respectively.

\subsection{Relocated AUX Component Configuration}

Routing density results for the configuration in Figure 8b shows the effect of moving the AUX component into the same watertight zone as the MAIN component. As expected, the move increased localized routing density near both AUX and MAIN components while decreasing the density in the region from which the AUX was removed from. The noticeable differences include an increase in routing density directly above AUX and the reduction of density above BH_4 (see Figure 6a) to $r_{i, j}=4.0$ from 5.0 .

Generally, changing the AUX location decreases the routing density variance across adjacencies in the vessel, at the cost of increased density in a single zone. The observed decrease in variance is driven by the elimination of high density routings between $\mathrm{BH}_{-} 2$ and $\mathrm{BH}_{-} 4$ above the damage control deck. Additionally, the localized routing density increase in the zone with both AUX and MAIN components is mitigated by their co-location. Co-location eliminates the prime mover power system routing over BH_4 and allows the AUX-PR_MVR and MAIN-PR_MVR connections to share routings.

The observed changes in routing density are in large part caused by applying Equation 1, which only counts the types of systems routings on an

edge, not the total number of component pair routings. Regardless of the 
ship section AUX is in, the distribution of component locations ensure that electrical power is routing (red in Figure 6b) throughout the vessel. Thus, moving AUX only significantly affects local areas and edges with prime mover power system routings.

Comparing Figures 8a and 8b suggests that, aside from a localized change in density, the routings in the overall physical solution is largely unchanged by moving the AUX component. This is a different type of conclusion to that which would be gained from a system optimization approach. Optimized system generation would provide the lowest routing density in both configurations. This may be useful for evaluating what might be the best case design (for a minimum distributed system objective), but does not necessarily help the designer understand how changing the configuration will change the possible overall ship design characteristics.

The analysis results of the first two vessel configurations show the utility of the proposed method. However, both configurations are more similar to commercial vessels than naval vessels. Differences between naval physical solutions and the distribution of routing density discussed so far are indicative of a primary naval distributed system objective, that is survivability. Noticeably, the ring-bus system layout that is often seen in naval applications [37] is not present. This is a result of the vessel geometry model that prevents distributed systems from routing through the watertight bulkheads. The configuration in Figure 7c explores how allowing longitudinal routings with sufficient damage control isolation features below the damage control deck can model the design implications of a ring-bus.

\subsection{Ring-Bus Configuration}

Figure $8 \mathrm{c}$ shows the routing density results for the vessel configuration in $7 \mathrm{c}$, where the network is adjusted to allow routings along the lowest deck. The adjusted network assumes that all systems in the logical architecture are able to route through watertight ducts and doors at this deck. While this change does not reflect best-practices, it allows us to explore longitudinal system routings between components that are not present in the other configurations. The result is a redistribution of routing density from the area above the damage control deck to the new routing paths. While this is apparent in Figure $8 \mathrm{c}$ and meets the expected ring-bus design, the routing density changes illustrate an interesting aspect of the physical solution for this architecture.

Creating the new paths increases the mean routing density $\bar{r}$ to 2.49 from 2.17 in the baseline configuration. On the surface, this is surprising 
because the new paths are expected to alleviate the number of systems forced to route over the separating bulkheads. Because $r_{i, j}$ is an average across system types, the lower level adjacencies create opportunities for more shared routings without eliminating those that already existed. The exception to this is the prime mover power system which routes along the lowest deck in this configuration, removing the highest density regions of the vessel above BH_2 and BH_4. The overall result is a higher, but more even balance of density across the vessel, which is reflected in the reduction of $\sigma_{r}$ from 1.40 to 0.81 .

Implications of increased system routing density can be evaluated with the positive relationship between outfit density and required production and engineering labor described in [9]. The routing density results suggest that the ring-bus distributed system solution may be more difficult to design and produce than the alternative, separated configurations. This may be mitigated by the more uniform distributed of routing density, which eliminates areas of the vessel with all five types of system routings.

Analysis of the three vessel configurations demonstrate that the proposed network schema and L-PAT algorithm make a possible predictor of a significant element of outfit density - distributed systems routing density - readily available. Given this information, designers can restructure component layout, allow certain bulkhead penetrations, or plan for future design work. Regardless of the resulting design decisions, the designer now has a physical solution metric, a method to interrogate what drives changes in the metric, and physical solution instantiations which can be further evaluated.

\section{Conclusion}

This paper presents an early-stage design perspective and method for exploring how the logical and physical architectures of distributed ship service systems impact its physical solution. The proposed network schema can be applied at the initial vessel concept and developed throughout the design process without requiring excessive detail or problem definition. Additionally, the L-PAT algorithm can be used to translate vessel knowledge traditionally available early in the design process into a representation of the distributed systems' physical solution. Analysis of the solutions provides an efficient

method for generating new design information to facilitate developing and improving design requirements for multirole vessels. 
The utility of this perspective is demonstrated by evaluating distributed systems routing density in three different configurations of a notional concept vessel. The L-PAT algorithm is applied to convert the structural zone and system models into ensembles of physical solutions. The generated ensembles are then evaluated to find the probabilistic density of the physical solution between structural zones. The resulting metric, mean distributed systems routing density, is suggested as one predictor of outfit density between structural zones and illustrates the potential analysis this perspective and methodology provides.

Extensions to the routing density analysis include route weightings based on estimated component load, density by number of system connectivities, and localized complexity calculation. Variation in the vessel model can accommodate three-dimension geometries, higher fidelity geometries, and incorporate varying bulkhead permeability based on system routing or bulkhead location. Routings, which are restricted to single shortest paths, can be relaxed to include variation in the number of disjoint paths and random-walk paths. Regardless of the analysis preformed, the application of the L-PAT algorithm demonstrates the value of the proposed perspective.

Considering a stochastic set of feasible outcomes may reveal design relationships that would not be identified through traditional concept design or system optimization methods. Further, this approach is not limited to routing density. Generated physical solution ensembles can be evaluated using traditional approaches, such as vulnerability analyses, or novel network-based approaches to develop statistical models of requirement relationships. However, even in its most basic form, the L-PAT algorithm and supporting design perspective provide a powerful and extensible approach for investigating the design of distributed systems.

The authors hope that the perspective presented here provides designers with a reliable way forward when considering architectural design of distributed ship service systems in early-stage ship design. The relationship between logical architecture, physical architecture, and physical solutions affects all aspects of the vessel. Understanding this relationship sooner in the design process is a critical step in trade-off analysis and decision making which can have major impact on vessel performance. 


\section{Acknowledgment}

This research received funding from the U.S. Office of Naval Research, Grant No. N00014-15-1-2752 as well as the U.S. Department of Defense (DoD) through the National Defense Science \& Engineering Graduate Fellowship (NDSEG) Program.

\section{References}

[1] N. Doerry, The Evolution of the Electric Warship, Naval Engineers Journal 126 (1) (2014) 175-186.

[2] J. Piff, Development and Application of Electric Ship Technology Discussed at the IEEE Electric Ship Technology Symposium (ESTS), Naval Engineers Journal 125 (3) (2013) 47-50.

[3] B. Kassel, S. Cooper, A. Mackenna, Rebuilding the NAVSEA Early Stage Ship Design Environment, Tech. rep., DTIC Document, Arlington, Virginia (2010).

[4] J. Chalfant, Early-Stage Design for Electric Ship, Proceedings of the IEEE 103 (12) (2015) 2252-2266.

[5] N. Doerry, Zonal Ship Design, Naval Engineers Journal 118 (1) (2006) 39-53.

[6] N. Doerry, Designing electrical power systems for survivability and quality of service, Naval Engineers Journal 119 (2007) 25-34.

[7] T. A. Trapp, Shipboard Integrated Engineering Plant Survivable Network Optimization, Ph.D. thesis, Massachusetts Institute of Technology (2015).

[8] R. G. Keane, Reducing Total Ownership Cost: Designing Inside-Out of the Hull, ASNE Day 2011.

[9] R. G. Keane, L. Deschamps, S. Maguire, Reducing Detail Design and Construction Work Content by Cost-Effective Decisions in Early-Stage Naval Ship Design, Journal of Ship Production and Design 31 (3) (2015) $1-14$. 
[10] A. T. Dobson, Cost Prediction Via Quantitative Analysis of Complexity in U.S. Navy Shipbuilding, Ph.D. thesis, Massachusetts Institute of Technology (2014).

[11] A. Miroyannis, Estimation of Ship Construction Costs, Ph.D. thesis, Massachusetts Institute of Technology (2006).

[12] R. A. Dellsy, M. C. Parker, D. T. Rigterink, Multi-Scale, Interdisciplinary Systems Analysis for Naval Platforms, Naval Engineers Journal 2 (127) (2015) 93-100.

[13] A. R. Greig, J. Coombes, D. J. Andrews, R. P. Pawling, Modelling The Heat Distribution In A Warship, in: World Maritime Technology Conference 2009, Mumbai, India, Janruary 2009, 2009, pp. 1-9.

[14] C. P. F. Shields, D. T. Rigterink, D. J. Singer, The Information Dual Network and its Applications to Naval Architecture, in: 12th International Marine Design Conference, Vol. 2, Tokyo, Japan, May 2015, 2015, pp. 1-8.

[15] C. P. F. Shields, M. J. Sypniewski, D. J. Singer, Understanding the relationship between naval product complexity and on-board system survivability using network routing and design ensemble analysis, in: International Symposium on Practical Design of Ships and Other Floating Structures 2016, Copenhagen, Denmark, September 2016, 2016, pp. 219-225.

[16] A. Brown, J. Waltham-Sajdak, Still Reengineering the Naval Ship Concept Design Process, Naval Engineers Journal 1 (127) (2015) 49-61.

[17] D. J. Andrews, Ship Project Managers Need to be Systems Architects not Systems Engineers, in: Maritime Project Management, London, United Kingdom, February 2016, 2016.

[18] D. T. Rigterink, Methods for Analyzing Early Stage Naval Distributed Systems Designs, Employing Simplex, Multislice, and Multiplex Networks, Ph.D. thesis, University of Michigan (2014).

[19] D. Rigterink, R. Piks, D. J. Singer, The use of network theory to model disparate ship design information, in: 12th Symposium on Practical Design of Ships and Floating Structures, Changwon City, Korea, October 2013, 2013. 
[20] D. J. Andrews, Marine Design - Requirement Elucidation Rather Than Requirement Engineering, in: International Marine Design Conference 2003, Athens, Greece, May 2003, 2003.

[21] D. J. Andrews, Marine Requirements Elucidation and the Nature of Preliminary Ship Design, International Journal of Maritime Engineering 153 (2011).

[22] D. J. Andrews, Art and science in the design of physically large and complex systems, Proceedings of the Royal Society A: Mathematical, Physical and Engineering Sciences 468 (2012) 891-912.

[23] S. K. Chandrasegaran, K. Ramani, R. D. Sriram, I. Horváth, A. Bernard, R. F. Harik, W. Gao, The evolution, challenges, and future of knowledge representation in product design systems, CAD Computer Aided Design 45 (2) (2013) 204-228.

[24] A. M. Cramer, S. D. Sudhoff, E. L. Zivi, Performance Metrics for Electric Warship Integrated Engineering Plant Battle Damage Response, IEEE Transactions on Aerospace and Electronic Systems 47 (1) (2011) 634646.

[25] R. Fang, W. Jiang, J. Khan, R. Dougal, System-Level Thermal Modeling and Co-simulation with Hybrid Power System for Future All Electric Ship, in: 2009 IEEE Electric Ship Technologies Symposium, IEEE, Baltimore, Maryland, April 2009, 2009, pp. 547-553.

[26] D. Mavris, D. DeLaurentis, Methodology for Examining the Simultaneous Impact of Requirements, Vehicle Characteristics, and Technologies on Military Aircraft Design, in: 22nd Congress of the International Council on the Aeronautical Sciences (ICAS), Harrogate, England, August 2000, 2000.

[27] D. Ullman, The mechanical design process, McGraw-Hill Higher Education, New York, 1992.

[28] J. W. Gillespie, A. S. Daniels, D. J. Singer, An Agent-based Framework for Simultaneously Arranging Spaces, Components, and Systems, in: 11th International Symposium on Practical Design of Ships and Other Floating Structures (PRADS 2010), Rio de Janeiro, Brazil, September 2010, 2010. 
[29] J. W. Gillespie, D. J. Singer, Helping bridge the gap between spaceonly arrangements and distributed system layouts, in: ASNE Day 2012, Arlington, Virginia, February 2012, 2012.

[30] J. M. Ross, A practical approach for ship construction cost estimating, in: COMPIT, Vol. 4, Siguenza, Spain, May 2004, 2004, pp. 98-110.

[31] D. G. M. Watson, A. W. Gilfillan, Some Ship Design Methods, Naval Architect 4 .

[32] J. Chalfant, B. Langland, S. Abdelwahed, C. Chryssostomidis, R. Dougal, A. Dubey, T. E. Mezyani, J. Herbst, T. Kiehne, J. Ordonez, S. Pish, E. Zivi, A Collaborative Early-Stage Ship Design Environment, CEM Publications (2012) 1-17.

[33] J. S. Chalfant, M. D. Ferrante, J. A. Noble, Interim Report on S3D/LEAPS Integration, Tech. rep., Massachusetts Institute of Technology. Sea Grant College Program (2014).

[34] R. A. Dougal, B. Langland, Catching it Early, in: Marine Technology, SNAME, 2016, pp. 65-71.

[35] E. R. Fiedel, J. S. Chalfant, C. Chryssostomidis, Cooling System EarlyStage Design Tool for Naval Applications, in: Proceedings of the 2011 Grand Challenges on Modeling and Simulation Conference, Society for Modeling \& Simulation International, The Hague, Netherlands, June 2011, 2011.

[36] J. Chalfant, M. Ferrante, C. Chryssostomidis, Design of a notional ship for use in the development of early-stage design tools, in: 2015 IEEE Electric Ship Technologies Symposium, ESTS 2015, IEEE, Alexandria, Virginia, June 2015, 2015, pp. 239-244.

[37] C. Chryssostomidis, C. M. Cooke, Space reservation for shipboard electric power distribution systems, in: 2015 IEEE Electric Ship Technologies Symposium, ESTS 2015, IEEE, Alexandria, Virginia, June 2015, 2015, pp. 187-192.

[38] D. J. Andrews, R. Pawling, SURFCON - A 21st Centrury Ship Design Tool, in: International Marine Design Conference 2003, Athens, Greece, May 2003, 2003. 
[39] D. J. Andrews, R. G. Pawling, The Application of Computer Aided Graphics to Preliminary Ship Design, in: International Marine Design Conference 2006, Ann Arbor, Michigan, May 2006, 2006, pp. 99-119.

[40] F. Mistree, W. Smith, B. Bras, J. Allen, D. Muster, Decision-based Design: A Contemporary Paradigm for Ship Design, Transactions, Society of Naval Architects and Marine Engineers 98 (1990) 565-597.

[41] Government Accountability Office, High Levels of Knowledge at Key Points Differentiate Commercial Shipbuilding from Navy Shipbuilding, Tech. Rep. GAO-09-322, Government Accountability Office (2009).

[42] NAVSEA, Ship Design Manager (SDM) and Systems Integration Manager (SIM) Manual (2012).

[43] D. J. Andrews, Two systems engineering models and their relevance to marine systems, in: The Systems Engineering Conference, London, United Kingdom, March 2012, 2012, pp. 1-11.

[44] R. Pawling, D. J. Andrews, Design Sketching - The Next Advance in Computer Aided Preliminary Ship Design?, in: COMPIT, Berlin, Germany, May 2011, 2011, pp. 426-441.

[45] G. Pitts, Computer-aided design: use and misuse, Computer-Aided Design 2 (4) (1970) 41-45.

[46] A. S. Daniels, F. Tahmasbi, D. J. Singer, Intelligent Ship Arrangement (ISA) Methodology Improvements and Capability Enhancements, in: International Marine Design Conference 2009, Trondheim, Norway, May 2009, 2009.

[47] M. G. Parsons, H. Chung, E. Nick, A. Daniels, S. Liu, J. Patel, Intelligent Ship Arrangements: A New Approach to General Arrangement, Naval Engineers Journal 120 (3) (2008) 51-65.

[48] A. S. Daniels, F. Tahmasbi, D. J. Singer, Intelligent Ship Arrangement Passage Variable Lattice Network Studies and Results, Naval Engineers Journal 122 (2) (2010) 107-119.

[49] A. S. Daniels, M. C. Parker, D. J. Singer, Intelligent Ship Arrangements (ISA) Passage Variable Lattice Network Templating Application, in: COMPIT, Berlin, Germany, May 2011, 2011, pp. 19-30. 
[50] J. W. Gillespie, A Network Science Approach to Understanding and Generating Ship Arrangements in Early-Stage Design, Ph.D. thesis, University of Michigan (2012).

[51] J. W. Gillespie, A. S. Daniels, D. J. Singer, Generating functional complex-based ship arrangements using network partitioning and community preferences, Ocean Engineering 72 (2013) 107-115.

[52] D. J. Andrews, Creative Ship Design, Transactions of the Royal Institution of Naval Architects.

[53] M. C. Parker, A Contextual Multipartite Network Approach to Comprehending the Structure of Naval Design, Ph.d. thesis, University of Michigan (2014).

[54] M. C. Parker, D. J. Singer, Analyzing the dynamic behavior of marine design tools using network theory, Ocean Engineering 106 (2015) 227237.

[55] C. P. F. Shields, D. C. Brefort, M. C. Parker, D. J. Singer, Adaptation of Path Influence Methodology for Network Study of Iteration in Marine Design, in: 12th International Maritime Design Conference, Vol. 2, Tokyo, Japan, May 2015, 2015, pp. 43-56.

[56] B. J. van Oers, A Packing Approach for the Early Stage Design of Service Vessels, Ph.d. thesis, Technical University of Delft (2011).

[57] B. J. van Oers, G. van Ingen, D. Stapersma, An integrated approach for the design of survivable ship services systems, in: International Naval Engineering Conference (INEC) 2012, Edinburgh, Scotland, May 2012, 2012 .

[58] M. E. J. Newman, The structure and function of complex networks, SIAM Review 45 (2) (2003) 167-256.

[59] M. E. J. Newman, Networks: an introduction, Oxford University Press, Oxford, 2010.

[60] S. Gómez, A. Díaz-Guilera, J. Gómez-Gardeñes, C. J. Pérez-Vicente, Y. Moreno, A. Arenas, Diffusion dynamics on multiplex networks, Physical Review Letters 110 (2) (2013) 1-5. 
[61] E. W. Dijkstra, A note on two problems in connection with graphs, Numerische Mathematik 1 (1) (1959) 269-271.

[62] P. J. Usher, A. L. Dorey, A Family of Warships, RINA Transactions 124 (1982). 